\section{Ältere Menschen: Tägliche Prothesenreinigung beugt Lungenentzündungen vor}

Kosuma T et al. Infrequent denture cleaning increased the risk of pneumonia among community-dwelling older adults: a population-based cross-sectional study. Sci Rep 2019; 9: 13734

Die Wirksamkeit der Mundpflege zur Prävention von Pneumonien in Pflegeheimen und Krankenhäusern ist bekannt. Menschen höheren Alters, die mit Unterstützung selbstständig wohnen, sind sich der Rolle der Prothesenreinigung zur Pneumonievorbeugung jedoch weiterhin nicht bewusst. Japa- nische Medizinsoziologen untersuchten den Zusammenhang zwischen einer seltenen Reinigung von Prothesen und dem Risiko einer Lungenentzündung bei dieser Personengruppe.

Die Ergebnisse dieser Querschnittsstudie basieren auf der Auswertung einer Umfrage der Japan Gerontological Evaluation Study (JAGES) des Jahres 2016. In dieser Umfrage wurden Erwachsene im Alter $\geq 65$ Jahre, die selbstständig und nicht in einer Pflegeeinrichtung lebten, zu sozialen, Verhaltens- und Gesundheitsthemen befragt. Der Fragebogen wurde per Post verschickt und auf gleichem Wege zurückgeschickt.

Als abhängige Variable wurde nach einer Lungenentzündung gefragt. Die Häufigkeit der Prothesenreinigung wurde als unabhängige Variable erfragt und als Kovariablen Alter, Geschlecht, Raucherstatus, Bildungsstatus, Einkommen, Anzahl der Zähne und Aktivitäten des täglichen Lebens (ADL), Komorbidität im Zusammenhang mit Schlaganfall oder Demenz und Erfahrung mit Pneumokokkenimpfungen innerhalb der letzten 5 Jahre dokumentiert.

Insgesamt bestand die Zielpopulation aus knapp 280000 Frauen und Männern, von denen 180000 Personen den Fragebogen zurückschickten (Rücklaufquote: 70,2\%). Davon verwendeten knapp 89000 (49,4\%) herausnehmbare Prothesen und wurden in diese Analyse einbezogen. In die Auswertung gelangten schließlich mehr als 70000 Teilnehmer. Das Durchschnittsalter lag bei 75,2 $\pm 6,5$ Jahren, 48,3\% waren männlich. Insgesamt hatten 2,3\% der Teil- nehmer innerhalb des letzten Jahres eine Lungenentzündung gehabt und 97,7\% keine. Eine Pneumonie war häufiger bei den Personen, die ihre Prothesen nicht täglich reinigten, insbesondere bei Personen, die $\geq 75$ Jahre alt waren. In dieser Gruppe hatten $2,9 \%$ derjenigen, die ihre Prothesen täglich reinigten, eine Lungenentzündung, aber in der Subgruppe derjenigen, die sie nicht täglich säuberten, waren dies $4,3 \%$.

Um einen möglichen Bias hinsichtlich der Kovariablen auszuschließen, erfolgte ein Propensity Score Matching hinsichtlich der Prothesenreinigung unter Verwendung des Imputationsverfahrens, d. h. der Ergänzung fehlender Daten, von MICE (Multiple iterated chained Equations). Dabei zeigte sich, dass alle Kovariablen zwischen denen, die ihre Prothesen täglich gereinigt hatten, und denjenigen, die dies nicht getan hatten, gut ausbalanciert waren. Demnach ergab sich durch die gelegentliche Reinigung der Zahnprothesen ein signifikanter Zusammenhang mit einer Pneumonie mit einer Wahrscheinlichkeit (Odds Ratio) von 1,30 (95\%-KI 1,01-1,68).

FAZIT

Die Ergebnisse dieser Studie belegen, dass die tägliche Reinigung von Prothesen die Eintrittswahrscheinlichkeit einer Lungenentzündung bei älteren Erwachsenen, die mit Unterstützung selbstständig leben, reduzieren könnte, so die Autoren.

Richard Kessing, Zeiskam 